\title{
Proyecto OIEA IWAVE en América Latina en apoyo del ODS 6: agua segura para todos de manera sostenible
}

\author{
Emilia Bocanegra \\ Universidad Nacional de Mar del Plata. IGCyC-CIC. Funes 3350.Mar del Plata, Argentina \\ emilia.bocanegra@gmail.com
}

\begin{abstract}
RESUMEN
La metodología IWAVE del Organismo Internacional de Energía Atómica (OIEA) para mejorar la disponibilidad y sostenibilidad del agua a través de evaluaciones integrales de los recursos hídricos nacionales propone fortalecer las capacidades de los países para recopilar, gestionar e interpretar datos hidrológicos y el uso de herramientas y técnicas avanzadas para mejorar la gestión de recursos. El OIEA llevó a cabo en América Latina el Proyecto ARCAL RLA 7018 (2014-2017) “Mejora del conocimiento de los recursos de aguas subterráneas para contribuir a su protección, gestión integrada y gobernanza" aplicando IWAVE. A partir de los resultados auspiciosos, el OIEA puso en marcha la segunda fase de IWAVE en la región, el Proyecto RLA 7024 (2018-2021) “Integración de la hidrología isotópica en las evaluaciones nacionales de los recursos hídricos". Los principales resultados incluyen: a) conocimiento de los tomadores de decisiones sobre la importancia de la información hidrológica sólida para mejorar la gestión de los recursos hídricos, b) el establecimiento de redes de monitoreo de isótopos y el uso de herramientas isotópicas para mejorar los modelos conceptuales hidrogeológicos, c) el desarrollo de escenarios de cambio climático y su impacto en los recursos hídricos, d) nuevas bases de datos hidrológicos nacionales, e) desarrollo/ implementación de Agendas Nacionales del Agua. Ambos proyectos han vinculado los datos hidrológicos con la toma de decisiones, en apoyo del Objetivo del Desarrollo Sostenible (ODS) 6 "Agua segura para todos de manera sostenible", en particular con ODS 6.5, que se enfoca en la gestión integrada de los recursos hídricos.
\end{abstract}

Palabras clave: aguas subterráneas, gestión, hidrología isotópica, América Latina.

\section{The IWAVE Project in Latin America in support of SDG 6: secure water for all in a sustainable way}

\begin{abstract}
The IWAVE methodology of the International Atomic Energy Agency (IAEA) to improve water availability and sustainability through comprehensive assessments of national water resources proposes to strengthen national capacities to collect, manage and interpret hydrological data and the use of advanced tools and techniques to improve the management of water resources. The IAEA carried out in Latin America the Project ARCAL RLA 7018 (2014-2017) "Improvement of knowledge of groundwater resources to contribute to its protection, integrated management and governance" using IWAVE. Based on the favourable results, the IAEA launched the second phase of IWAVE in the region, Project RLA 7024 (2018-2021) "Integration of isotope hydrology in national assessments of water resources."The main results include: a) knowledge for decision-makers on the importance of sound hydrological information to improve the management of water resources; $b$ ) the establishment of isotope monitoring networks and the use of isotopic tools to improve conceptual hydrogeological models; $c$ ) the development of scenarios of climate change and their impact on water resources; d) new national hydrological databases; and e) the development/implementation of National Water agendas. Both projects have linked hydrological data with decision-making in support of the sustainable development goal (SDG) 6 "Safe water for all in a sustainable way", in particular with SDG 6.5, which focuses on integrated water resource management.
\end{abstract}

Keywords: groundwater, management, isotope hydrology, Latin America. 


\section{Introducción}

América Latina concentra la tercera parte de los recursos hídricos renovables del mundo, que idealmente deberían satisfacer la demanda de su población, que representa el $9 \%$ de la población mundial. Sin embargo, 25 millones de personas no tienen acceso a agua segura (UNESCO, 2019).

Los recursos de agua subterránea en muchas partes de América Latina son la principal o única fuente de agua, proporcionando servicios de suministro urbano, desarrollo de las actividades productivas y mantenimiento de los ecosistemas. Los beneficios que el agua subterránea proporciona al bienestar humano son, sin embargo, afectados por el aumento progresivo de la demanda y una disminución en la calidad y disponibilidad de agua subterránea.

Los principales impulsores directos de cambios de los servicios de disponibilidad de aguas subterráneas son la extracción intensiva, la baja recarga, la escasez y la falta de infraestructura. Los principales impulsores directos de cambios en los servicios de calidad de las aguas subterráneas están asociados con la agricultura, la ganadería, la industria, la minería, la falta de saneamiento, la eliminación de desechos y los procesos naturales. Estos factores, en ausencia de un sistema de gestión suficientemente sólido, pueden generar desequilibrios entre la oferta y la demanda en el mediano o largo plazo, con riesgos para la producción inaceptables.

En ocasiones existen inconsistencias, vacíos o insuficiencias en los instrumentos legales previstos en la institucionalidad para responder a las necesidades de desarrollo de los recursos hídricos o de su regulación. Áreas que presentan frecuentemente un insuficiente desarrollo o vacíos se refieren a aguas subterráneas, ordenamiento territorial, regulación de los aspectos ambientales y de la calidad de las aguas y contaminación difusa, entre otras (CEPAL, 2018).

Se han hecho muchos esfuerzos para resolver estos problemas, pero no de una manera coordinada y eficiente, debido a deficiencias en el conocimiento integral de los recursos hídricos a nivel regional.

EI Organismo Internacional de Energía Atómica (OIEA) está implementando desde 2012 el Proyecto de Mejora de la Disponibilidad de Agua (IWAVE: Water Availability Enhancement Project), dirigido a los Estados miembros para mejorar la disponibilidad y la sostenibilidad del agua dulce (con énfasis en las aguas subterráneas) a través de evaluaciones integrales de los recursos hídricos nacionales (Dunning, 2010).

IWAVE fue el resultado de algunas situaciones técnicas clave:

- Existencia de proyectos con estudios isotópicos desconectados de la hidrología convencional, con resultados pobres y de bajo valor.

- Comprensión inadecuada de los sistemas hidrológicos y de los enfoques de gestión.

- Esfuerzos del OIEA durante muchos años en apoyar proyectos locales desarrollados por instituciones de investigación, que no se traducían en una mejora de la gestión de los recursos hídricos del país.

- Colaboración limitada por parte de las autoridades nacionales del agua y entre los Estados miembros en cuestiones transfronterizas.

IWAVE se aplicó inicialmente en tres países piloto, Costa Rica, Filipinas y Omán obteniendo importantes resultados en la evaluación de los recursos hídricos nacionales (IAEA, 2013).

Los logros en Costa Rica fueron la contribución al Plan Nacional de Gestión Integrada de Recursos Hídricos, la creación de un sistema de información hidrológica centralizado, un balance hídrico a nivel nacional, la elaboración de planes de gestión y protección para zonas de recarga, un programa de muestreo integral de aguas superficiales y subterráneas para análisis de isótopos y el desarrollo de mapas temáticos de línea de base de isótopos a escala nacional.

En Filipinas la experiencia ganada por la Junta Nacional de Recursos Hídricos en la gestión del agua subterránea, a través de la metodología IWAVE, llevó a su designación como agencia principal para la gestión nacional de aguas subterráneas, responsable de planes y objetivos nacionales del ODS 6.4, se realizaron nuevas inversiones en modelación de aguas subterráneas y GIS, se mejoraron los métodos y capacidades para interpretar datos e información produciendo nuevos análisis del balance hídrico y el cálculo del flujo base como práctica estándar de gestión del agua, se realizaron evaluaciones de aguas subterráneas y de vulnerabilidad en 9 cuencas bajo estrés hídrico con mandato para la mitigación, planificación y regulación y se amplió el monitoreo del agua subterránea a nivel nacional.

En Omán se estableció la Red de Monitoreo del Agua Subterránea que incluye la generación de datos sobre 127 cuencas hidrográficas para la Base Nacional de datos de agua subterránea, se establecieron estaciones de medición de precipitaciones, se contribuyó con el Proyecto Nacional de Balance de Agua y se realizaron estudios de caracterización y vulnerabilidad de acuíferos priorizados.

En el período 2014-2017, el OIEA llevó a cabo el Proyecto RLA 7018 "Mejora del conocimiento de los recursos de aguas subterráneas para contribuir a su protección, gestión integrada y gobernanza", que se propuso el fortalecimiento de las capacidades nacionales para realizar evaluaciones integradas de los recursos hídricos en América Latina. El proyecto se desarrolló bajo los auspicios de ARCAL, un acuerdo de cooperación para promover la ciencia y la tecnología nucleares en la región.

A partir de los resultados auspiciosos, el OIEA puso en marcha, en el período 2018-2021, el Proyecto RLA 7024 “Integración de la hidrología isotópica en las evaluaciones nacionales de los recursos hídricos", que constituye la segunda fase de aplicación de IWAVE en la región.

Entre 2012 y 2017 el OIEA implementó en África, en 
la región del Sahel, un proyecto regional para ayudar a administrar los recursos hídricos compartidos, en el que se proporcionó equipamiento y capacitación a científicos de 13 países y se estudiaron y cartografiaron cinco sistemas de acuíferos transfronterizos (Mikhailova, 2019). Sobre la base de este proyecto e inspirado en la experiencia en la aplicación de IWAVE del proyecto regional latinoamericano RLA 7018, en 2018 el OIEA inició, con aplicación de esta metodología, un proyecto regional africano en los países del Sahel, siendo las áreas piloto Benin, Camerún, Ghana, Níger y Nigeria, durante el cual se espera abordar las brechas de capacidad e información restantes en hidrología nacional, de modo de contribuir a una mejor gestión en el sector del agua.

El proyecto IWAVE en América Latina ha vinculado los datos hidrológicos con la toma de decisiones en apoyo del Objetivo de Desarrollo Sostenible (ODS) 6, que propende a "Asegurar agua para todos de forma sostenible", en particular del 6.5 relacionado con la gestión integrada de los recursos hídricos.

El objetivo del trabajo es presentar desde un enfoque regional los resultados de la aplicación de IWAVE en algunos países de América Latina y las lecciones aprendidas, a fin de realizar un aporte a la implementación de la metodología en el futuro, en otros países de la región.

\section{El agua como objetivo transversal vinculado con la ODS}

La Asamblea General de Naciones Unidas aprobó en 2015 la Agenda 2030, cuyos ODS reflejan la aspiración global de transformar el mundo antes del año 2030 e incluye 17 compromisos principales (PNUD, 2015), que abarcan desde poner fin a la pobreza y al hambre hasta promover la igualdad de género y el crecimiento económico.

La ONU propone alcanzar el ODS 6 "Garantizar la disponibilidad y la gestión sostenible del agua y el saneamiento para todos", a través del logro de diversas metas para 2030, tales como el acceso universal y equitativo al agua potable, el acceso equitativo a servicios de saneamiento, la mejora de la calidad del agua mediante la reducción de la contaminación, el aumento del uso eficiente de los recursos hídricos en todos los sectores, la gestión integrada de los recursos hídricos y la protección de los ecosistemas relacionados con el agua, ampliando la cooperación internacional para la creación de capacidades y fortaleciendo la participación de las comunidades locales en la mejora de la gestión del agua.

Los ODS reconocen la importancia de las cuestiones relacionadas con el agua por derecho propio y como requisito para hacer realidad muchos de los otros objetivos. El derecho humano al agua es el derecho de todos a disponer de agua suficiente, salubre, aceptable, accesible y asequible para el uso personal y doméstico.

El agua es la base del desarrollo sostenible. La reducción de la pobreza, el crecimiento económico y la sostenibilidad ambiental se sustentan en los recursos hídricos y en la gama de servicios que proporcionan. Desde la alimentación y la seguridad energética hasta la salud humana y ambiental, el agua contribuye a mejorar el bienestar social y el crecimiento inclusivo, lo cual afecta a la subsistencia de miles de millones de seres humanos.

El agua es un objetivo transversal vinculado a los objetivos de desarrollo de las sociedades, los cuales

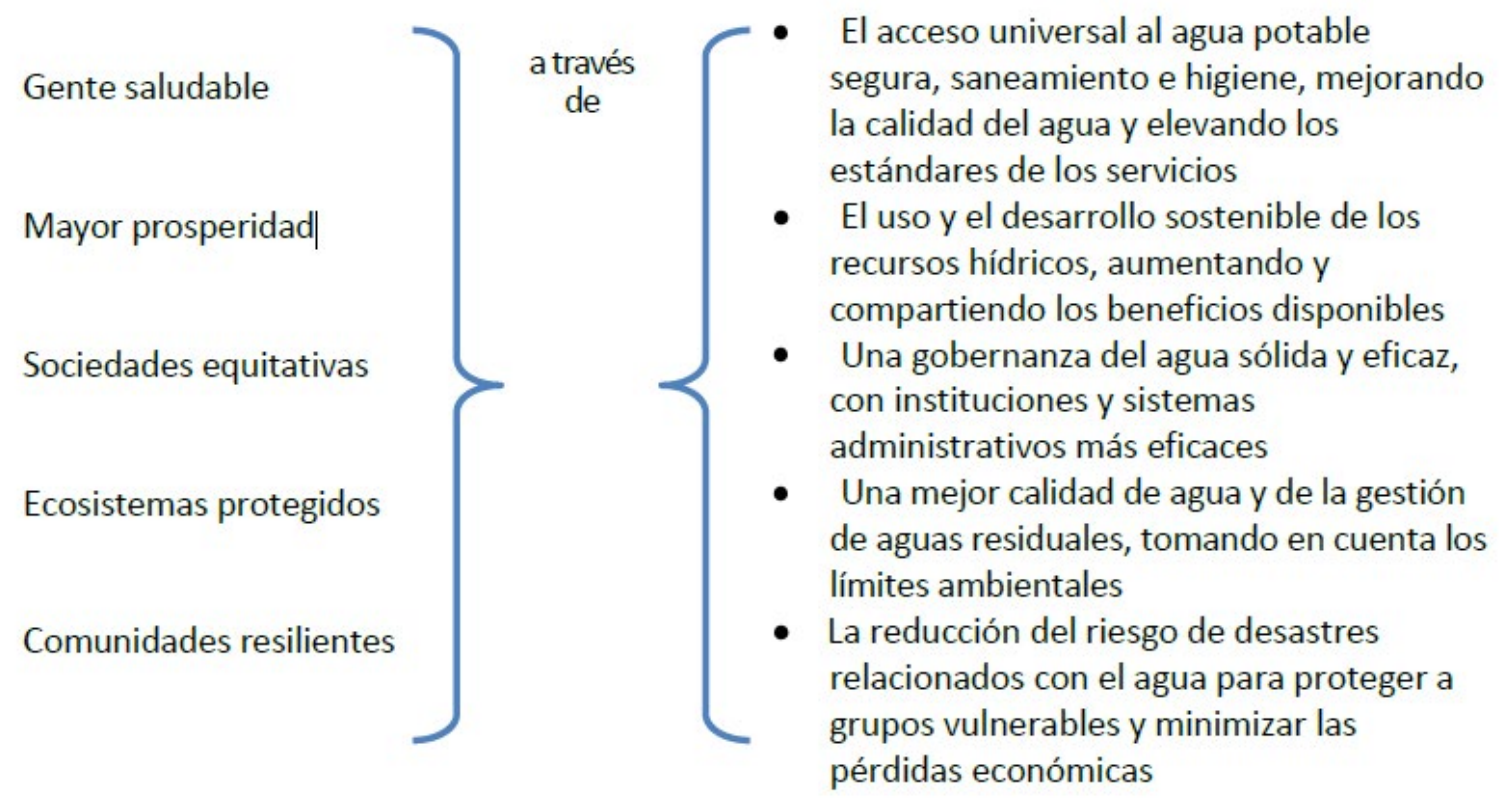

Figura 1. Resultados de desarrollo a partir de los ODS vinculados con el agua (UN-WATER, 2015).

Figure 1. Development results from the SDGs linked to water (UN-WATER, 2015). 


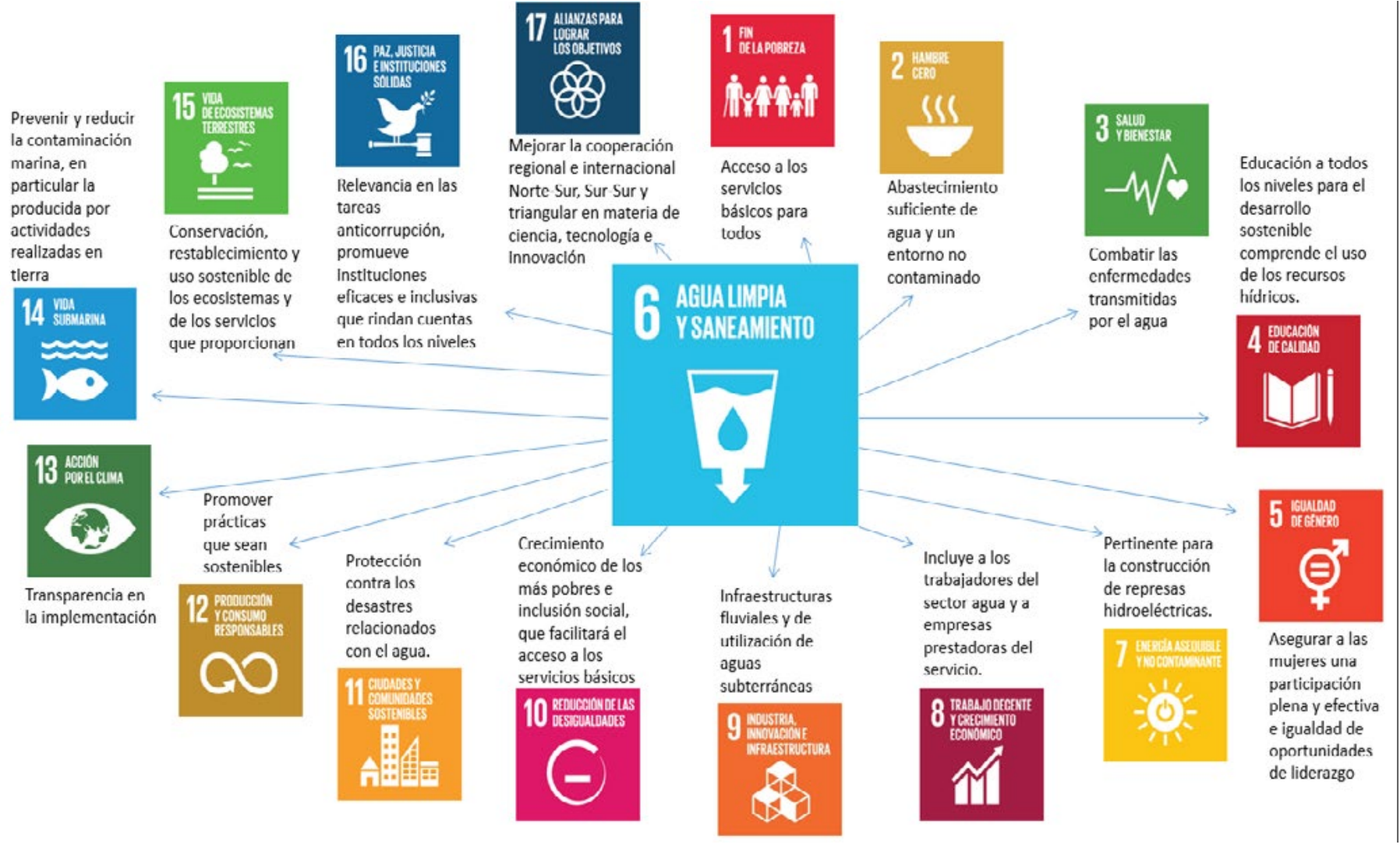

Figura 2. Transversalidad del ODS 6 en relación a los otros ODS (Fuente: elaboración propia).

Figure 2. Transversality of SDG 6 in relation to the other SDGs (Source: own elaboration).

promueven la dignidad humana y aseguran que los logros sean sostenibles a largo plazo (UN-WATER, 2015) (Figura 1).

Los ODS están interrelacionados y muchos dependen especialmente de la gobernanza y la gestión eficaces del agua para su uso en fines alimentarios, para proteger el medio ambiente y para mejorar la calidad de vida de las personas (Figura 2).

La clave es avanzar en amplios objetivos de sostenibilidad sin causar impactos adversos y daños en otras partes del sistema. Esto requiere ciencia integrada, pensamiento e instituciones integradoras. Este enfoque es consistente con la estrategia utilizada para definir los ODS, que proporcionan un conjunto integral e integrado de objetivos de sostenibilidad global (Gleick, 2017).

\section{Metodología}

IWAVE surge como un proceso estructurado, integral y colaborativo que a través de la aplicación de la hidrología isotópica contribuye a mejorar la gestión de los recursos hídricos.

La metodología IWAVE consiste en los siguientes pasos (IAEA, 2012):

a. Identificar vacíos a nivel nacional/provincial en información y datos hidrológicos. b. Determinar la experiencia, la tecnología y la infraestructura requeridas para completar los vacíos identificados.

c. Formular e implementar la metodología óptima para la utilización de técnicas isotópicas.

d. Desarrollar una estrategia para la colaboración con otras organizaciones en orden de completar los vacíos técnicos e institucionales identificados

El Proyecto RLA 7018 contó con la participación de once países (Argentina, Bolivia, Brasil, Chile, Colombia, Cuba, Ecuador, México, Nicaragua, Paraguay y Venezuela), en los que se evaluó la disponibilidad de información hidrológica, la normativa legal y las políticas públicas (Bocanegra et al, 2016), y se abordó el fortalecimiento de capacidades a través de cursos de alcance regional o nacional. En cuatro áreas piloto: Argentina, Brasil, Ecuador y Nicaragua, en las que se establecieron compromisos al más alto nivel con las principales instituciones que participan en la gestión del agua de cada país, se implementó un plan de acción que incluyó la identificación y priorización de vacíos de conocimiento hidrológico y vacíos institucionales, la capacitación en temas específicos y el completado de vacíos priorizados. Argentina y Brasil llevaron a cabo a escala nacional el fortalecimiento de capacidades en hidrología isotópica y la caracteri- 
zación isotópica del agua de lluvia a través de la instalación de estaciones integradas a la GNIP (Global Network of Isotopes in Precipitation). Sin embargo, teniendo en cuenta su extensión geográfica, la identificación y completado de vacíos en la información hidrológica se llevó a cabo en un nivel inferior, la provincia de Buenos Aires $\left(307571 \mathrm{~km}^{2}\right)$ y el acuífero Urucuia (estados de Bahía, Maranhão, Piauí, Tocantins y Minas Gerais, $325954 \mathrm{~km}^{2}$ ), respectivamente. Ecuador $\left(283560 \mathrm{~km}^{2}\right)$ y Nicaragua $\left(129494 \mathrm{~km}^{2}\right)$ ejecutaron el proyecto a nivel nacional.

El Proyecto RLA 7024 cuenta con la participación de quince países (Argentina, Bolivia, Brasil, Chile, Colombia, Costa Rica, Cuba, Ecuador, México, Nicaragua, Panamá, Paraguay, República Dominicana y Venezuela), continúa con el fortalecimiento de las capacidades en conocimiento hidrológico y gestión de los recursos hídricos de la región, y ha comenzado la implementación de la metodología IWAVE en cuatro escenarios piloto: Bolivia (6 Departamentos), Colombia (jurisdicciones de 11 Corporaciones Autónomas Regionales), México (3 Cuencas) y Paraguay (2 Sistemas acuíferos).

\section{Resultados}

Conformación de Equipos de Gestión de Proyectos Los proyectos RLA 7018 y 7024 comenzaron las actividades con una misión de experto del OIEA a cada una de las áreas piloto para la identificación de las principales instituciones que deberían estar involucradas en los mismos y la revisión del plan de trabajo. Se realizaron talleres con la participación de gran número de instituciones vinculadas a la gestión y/o investigación de los recursos hídricos (un promedio de 10 instituciones en cada país), durante los cuales se pre- sentaron la misión, el ámbito de acción (nacional, provincial, departamental, municipal), las principales actividades y las capacidades técnicas e institucionales.

En cada taller se realizó una dinámica sobre interacción entre las instituciones con el objetivo de valorar el accionar de cada una de ellas, que consistió en presentar en diversos boxes las siglas de las instituciones presentes y unirlos por medio de flechas de acuerdo a los intercambios de datos, información y servicios propuestos por cada delegado. El resultado para Ecuador y Paraguay se muestra en la Fig. 3 a y b respectivamente. El diseño en forma de red de las interacciones ayudó a tomar conciencia de la importancia de desarrollar sinergias para mejorar la gestión de los recursos hídricos.

Como resultado de estos talleres se identificaron las instituciones que conformarían el equipo de gestión del proyecto, en general 4 en cada país, tratando de incluir 3 instituciones vinculadas a la gestión y una a la investigación de los recursos hídricos. Asimismo se realizaron visitas protocolares a las instituciones seleccionadas a fin de confirmar el compromiso de sus directivos en la ejecución del proyecto.

La figura 4 muestra la conformación de los equipos de gestión de los proyectos RLA 7018 y 7024 en los 8 países que constituyen las áreas piloto; se aprecia la participación entre las principales partes interesadas, de organismos de políticas públicas y de control, que ejercen su función como Autoridades del Agua o como dependencias dentro de Ministerios del Ambiente, las universidades, los institutos nacionales de investigación del agua y los operadores de servicios de agua, entre otros.

Las funciones de las principales partes interesadas son:

- Agencias Nacionales/Regionales de Agua: autoridades responsables de formular las políticas
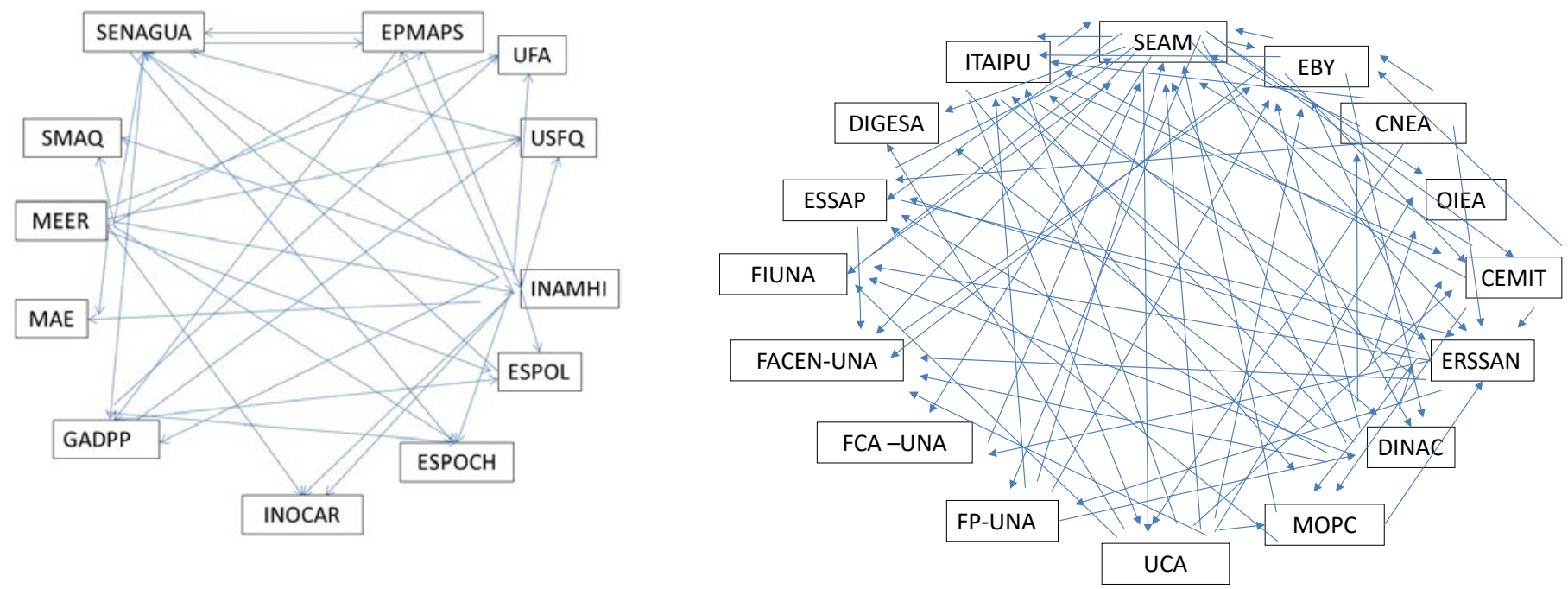

Figura 3. Dinámica sobre interacción entre instituciones vinculadas a la gestión y/o investigación de los recursos hídricos, realizada durante el taller para conformación del Equipo de gestión del proyecto, a) en Ecuador (Proyecto RLA 7018), b) en Paraguay (Proyecto RLA 7024). Figure 3. The dynamics of the interaction between institutions linked to the management and / or research of water resources carried out during the workshop for the formation of the Project Management Team, a) in Ecuador (Project RLA 7018), b) in Paraguay (Project RLA 7024). 
de agua y supervisar todas las actividades y trabajos relacionados con el estudio, recolección, uso, conservación y evacuación del agua.

- Ministerios de Medio Ambiente: responsables de promover la sostenibilidad ambiental del país mediante la conservación, protección, recuperación y aseguramiento de condiciones ambientales. En algunos países son autoridades del agua.

- Servicios geológicos: organismos nacionales encargados de generar, transferir y difundir los conocimientos científicos y tecnológicos sobre los recursos hídricos.

- Operadores de servicios: empresas públicas o privadas responsables de la provisión, administración, operación, mantenimiento, control, estudios, investigación y aplicación de nuevas tecnologías para el suministro de agua potable.

- Sector académico, ciencia y tecnología: universidades, institutos de investigación.

En cada país los equipos de gestión del proyecto mantienen reuniones periódicas para el seguimiento y actualización del plan de acción, poniendo énfasis en la organización de las capacitaciones técnicas e institucionales, en la generación de información hidrológica, en el fortalecimiento de las vinculaciones interinstitucionales y en la elaboración de lineamientos para la implementación de la Gestión Integrada de Recursos Hídricos (GIRH).

Identificación de brechas en la comprensión hidrológica, datos e información

La identificación de las brechas fue realizada durante la primera reunión de coordinación de cada proyecto, realizadas en Viena, Austria, con la participación de 4 representantes del más alto nivel de las instituciones que conforman el equipo del proyecto en cada país, es decir, 16 representantes en cada reunión. Posteriormente esta identificación de brechas fue consensuada en respectivos talleres nacionales, con la participación de profesionales técnicos además de los altos funcionarios miembros de los equipos de proyecto.

En el proyecto RLA 7018, los 4 países piloto realizaron un listado de las principales brechas. Si bien los países son muy heterogéneos en los aspectos de

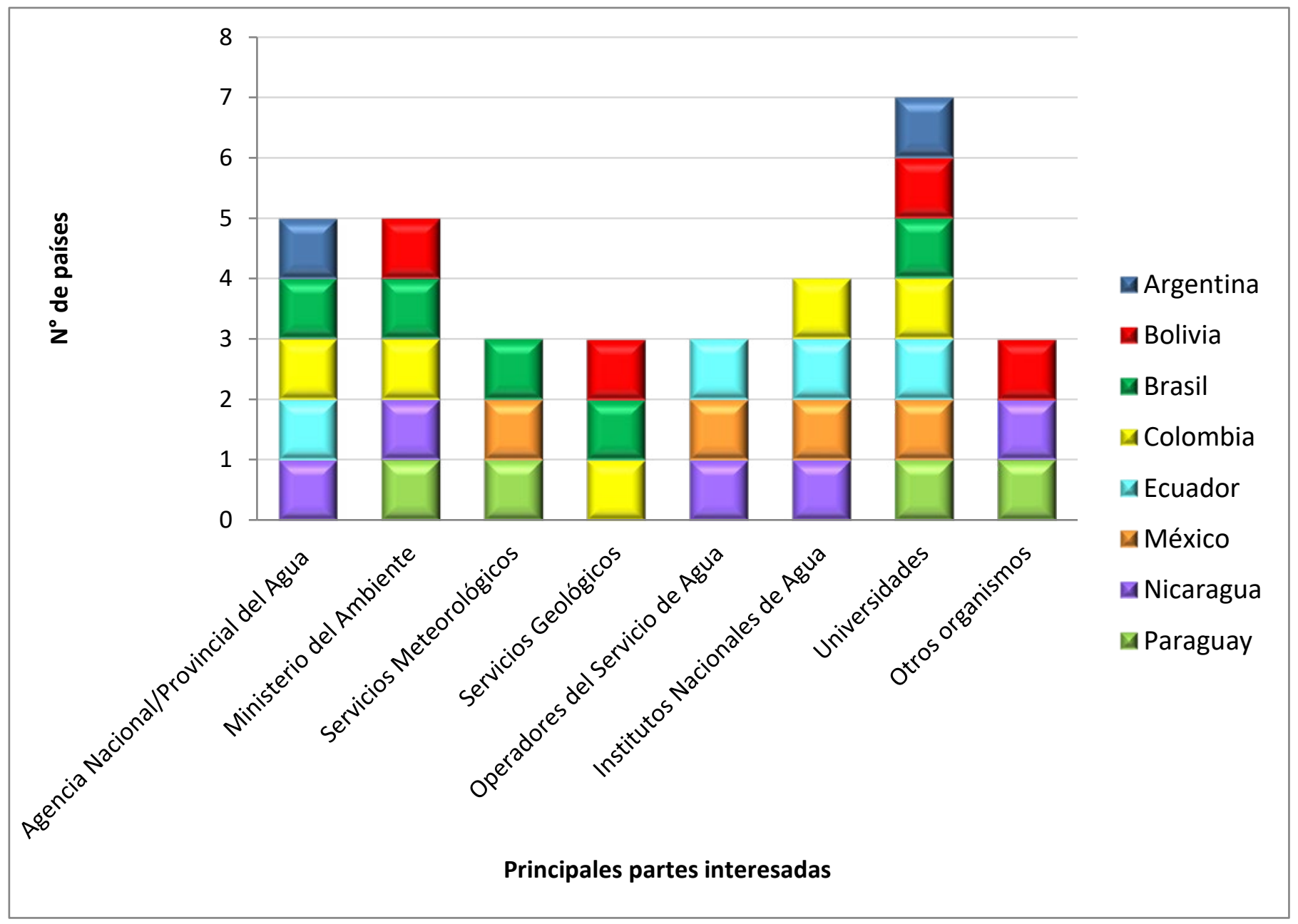

Figura 4. Instituciones que conforman los Equipos de Gestión de los Proyectos RLA 7018 y 7024.

Figure 4. Institutions that make up the Management Teams of the RLA 7018 and 7024 Projects. 
informaciones hidrológicas e incluso institucionales, estas brechas se pueden caracterizar en forma general como (Bocanegra et al, 2015):

a. Vacíos de conocimiento hidrometeorológico: deficiencias en la captura de datos e identificación y recopilación de información existente (incluyendo determinaciones isotópicas) en diferentes cuerpos de agua, insuficiente capacitación (con énfasis en herramientas isotópicas) de operadores de redes de muestreo, escasos laboratorios de análisis de agua certificados, escasos laboratorios de hidrología isotópica y ausencia de procesos de acreditación de los mismos, deficiencias en modelos conceptuales hidrológicos, limitado uso de modelos numéricos, falta de mapas hidrogeológicos unificados.

b. Vacíos de conocimiento de los usos de los recursos hídricos: deficiencias en la captura y validación de datos (caudales de extracción, niveles piezométricos, parámetros hidroquímicos, etc.).

c. Vacíos en la planificación y gestión de los recursos hídricos: debilidades institucionales, insuficiente desarrollo de un marco conceptual vinculado a la gestión integrada de los recursos hídricos y falta de modelos predictivos que contemplen el cambio global.
En el proyecto RLA 7024 se propuso una matriz para identificar y priorizar los vacíos, con el fin de contar con una herramienta más clara y que permitiera el análisis comparado del estado de situación del conocimiento en los diferentes países (Figura 4).

A partir de la identificación y priorización de los vacíos, los países piloto de los proyectos RLA 7018 y 7024 han establecido planes de acción para su completado.

\section{Fortalecimiento de capacidades institucionales $y$ técnicas}

La metodología IWAVE tiene un énfasis significativo en la capacitación en aspectos técnicos (incluyendo la hidrología isotópica) e institucionales a efectos de completar los vacíos identificados y así contribuir a la mejora efectiva de la gestión de los recursos hídricos; los principales destinatarios son profesionales pertenecientes a los organismos de gestión favoreciendo de esta manera el fortalecimiento de capacidades en dichas instituciones. La Figura 6 ilustra la distribución de estas actividades del RLA 7018 y la participación de los países en las mismas. Se realizaron varios cursos regionales así como cursos, talleres y asesorías en los países piloto del proyecto. El fortalecimiento de capacidades benefició a 520 personas de 15 países.

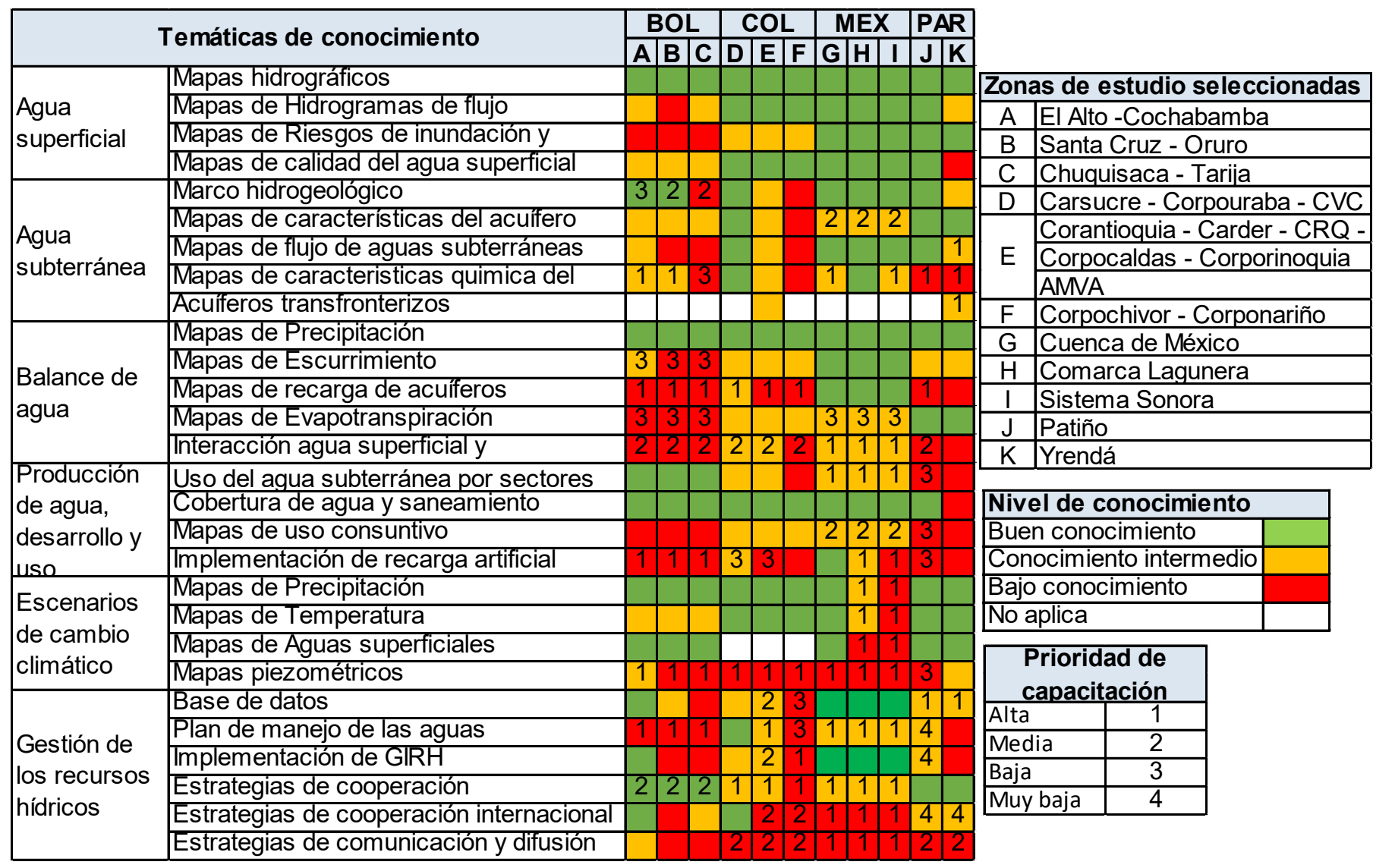

Figura 5. RLA 7024: Identificación y priorización de las principales brechas de conocimiento por país. Figure 5. RLA 7024: Identification and prioritization of the main knowledge gaps by country. 
EI OIEA ha proporcionado diversos suministros a los países para la ejecución de los proyectos, tales como sondas multiparamétricas, equipos de medición de parámetros fisicoquímicos para campo, colectores de agua de lluvia y espectrómetros láser para la determinación de isótopos estables del agua.

El Proyecto RLA 7024 en su primer año de ejecución ha realizado cursos regionales sobre Hidrología isotópica y sobre Uso de isótopos estables del agua en el contexto de cambio climático, y ha apoyado la capacitación integral en herramientas hidrológicas e hidrogeológicas relacionadas con la evaluación y gestión de los recursos hídricos, a través de la participación en cursos regionales organizados por otros organismos, beneficiando de esta manera a todos los países intervinientes en el proyecto. En las áreas piloto, ha apoyado talleres nacionales para facilitar y desarrollar el plan de acción del proyecto, ha realizado misiones de experto sobre aspectos institucionales, un curso sobre recarga artificial de acuíferos, un taller sobre GIRH y capacitación en la instalación de equipos de laboratorio para determinación de isótopos estables y de tritio.

\section{Completado de vacíos de conocimiento hidrológico $e$ institucionales $y$ caracterización preliminar hidrogeológica}

Los proyectos RLA 7018 y 7024 en cada área piloto sumaron sinergias con proyectos de instituciones de investigación o de gestión, o con proyectos nacionales del OIEA, permitiendo optimizar recursos y capacidades técnicas para facilitar la concreción de diversas actividades, tales como salidas de campañas, muestreos y determinaciones analíticas, confección de mapas e interpretación de resultados, entre otros.

La aplicación de la metodología IWAVE en las 4 áreas piloto del Proyecto RLA 7018 permitió completar vacíos de conocimiento hidrológico y realizar diversos estudios de caracterización hidrogeológica, con énfasis en hidroquímica e isotopía a nivel nacional o provincial. Entre ellos se destacan:

- Evaluación de la disponibilidad de datos hidrológicos

- Bases de datos hidroquímicos e isotópicos $\left({ }^{2} \mathrm{H}\right.$, ${ }^{18} \mathrm{O},{ }^{3} \mathrm{H}$ )

- Mapas de parámetros hidroquímicos e isotópicos

- Modelos conceptuales hidrogeológicos mejorados

- Mapas hidrogeológicos

- Mapas de uso de la tierra

- Desarrollo de escenarios de cambio climático y su impacto en los recursos hídricos

- Instalación de una red nacional de colectores para el monitoreo isotópico de la precipitación (GNIP)

Algunos ejemplos de los resultados obtenidos se muestran en las Figuras 7 y 8 . En el área piloto de Argentina se seleccionaron 5 cuencas prioritarias en el conurbano bonaerense teniendo en cuenta el estado de los recursos hídricos, la densidad poblacional e industrial, la fragilidad social y la condición hidrológica, y diversas cuencas del sudeste bonaerense de gran interés socioeconómico por sus actividades agrícolas, ganaderas y turismo. Se estableció la correlación de la información isotópica con las diferentes regiones hidrogeológicas de la provincia de Buenos Aires (Figura 7) y se elaboraron mapas de patrones de distribución de isótopos (Rives et al, 2018). Mediante el uso de datos isotópicos e hidroquímicos se recogió importante información sobre zonas de recarga, patrones de flujo, tiempo de residencia y tasa de renovación de agua subterránea, lo que constituye una herramienta valiosa para la toma de decisiones de la Autoridad del Agua.

Nicaragua, que entre sus principales brechas identificó el desconocimiento de la variación climática y su impacto en los recursos hídricos, lo que limitaba el diseño e implementación de planes preventivos para garantizar el abastecimiento de agua potable, salud, agricultura y generación de energía alternativa, realizó en el marco del Proyecto RLA 7018 la simulación de escenarios de cambio climático a nivel nacional y la evaluación del comportamiento de los recursos hídricos en cuencas específicas del país (Figura 8).

En lo referente a los vacíos en la planificación y gestión de los recursos hídricos, en los que se ponían de manifiesto las debilidades institucionales y el insuficiente desarrollo de un marco conceptual vinculado a la GIRH, el Proyecto RLA 7018 contribuyó con:

- Fortalecimiento de la cooperación interinstitucional entre organismos de gestión y de investigación, generación conjunta de datos e información y accesibilidad de bases de datos.

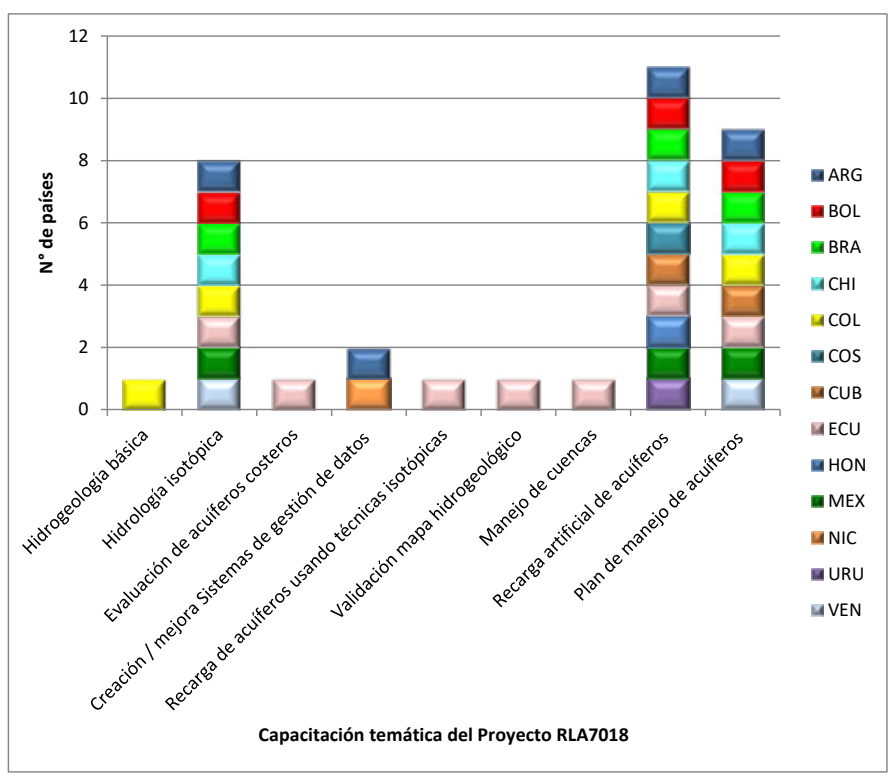

Figura 6. Aportaciones a los países a través de entrenamientos. Figure 6. Contributions to the countries through training. 


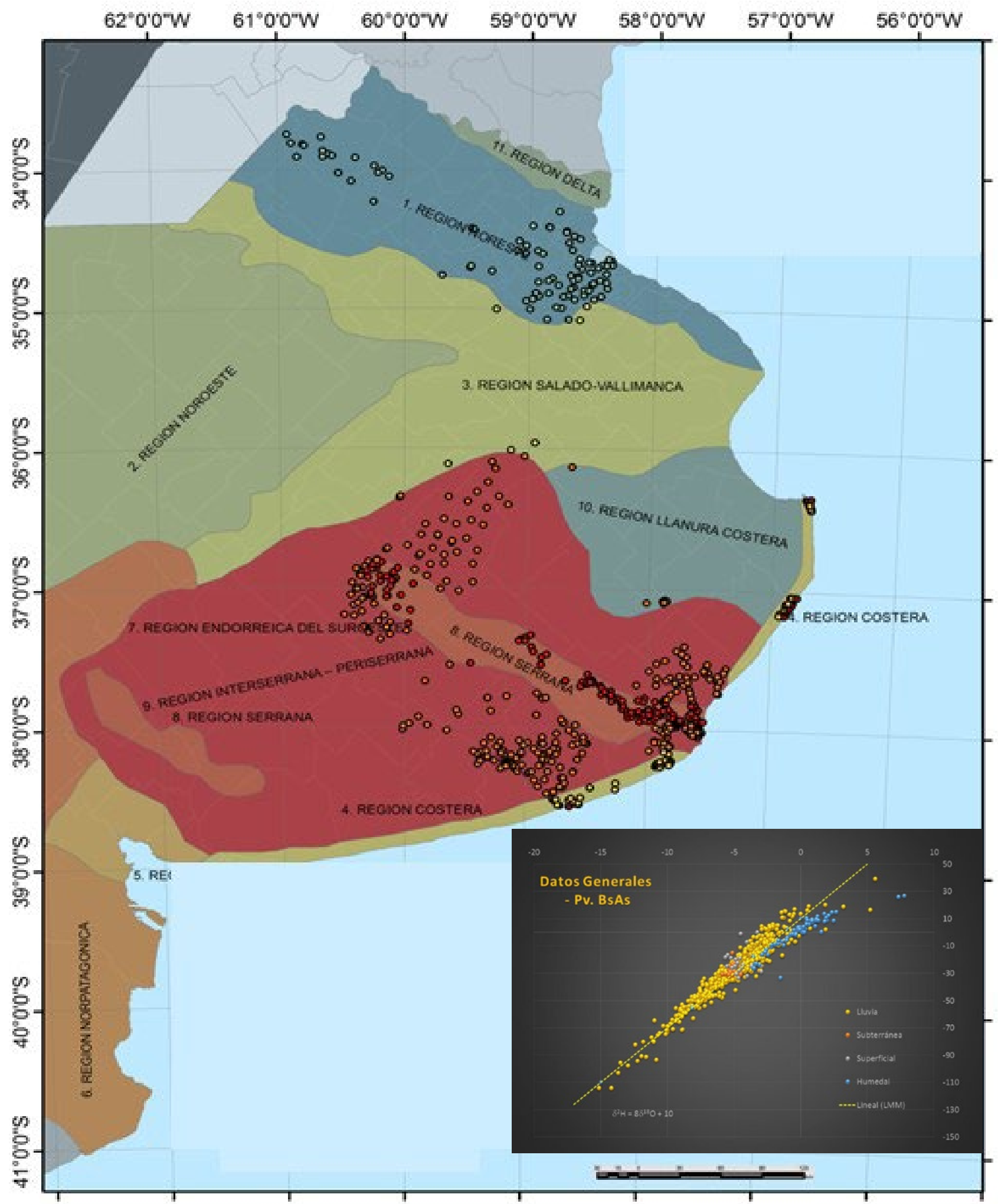

Figura 7. Mapa de regiones hidrogeológicas de la provincia de Buenos Aires y distribución de datos de valores isotópicos del agua subterránea en las áreas prioritarias del Proyecto RLA7018 (Rives et al, 2018).

Figure 7. Map of hydrogeological regions of the province of Buenos Aires and the distribution of isotopic values of groundwater in the priority areas of Project RLA7018 (Rives et al, 2018). 


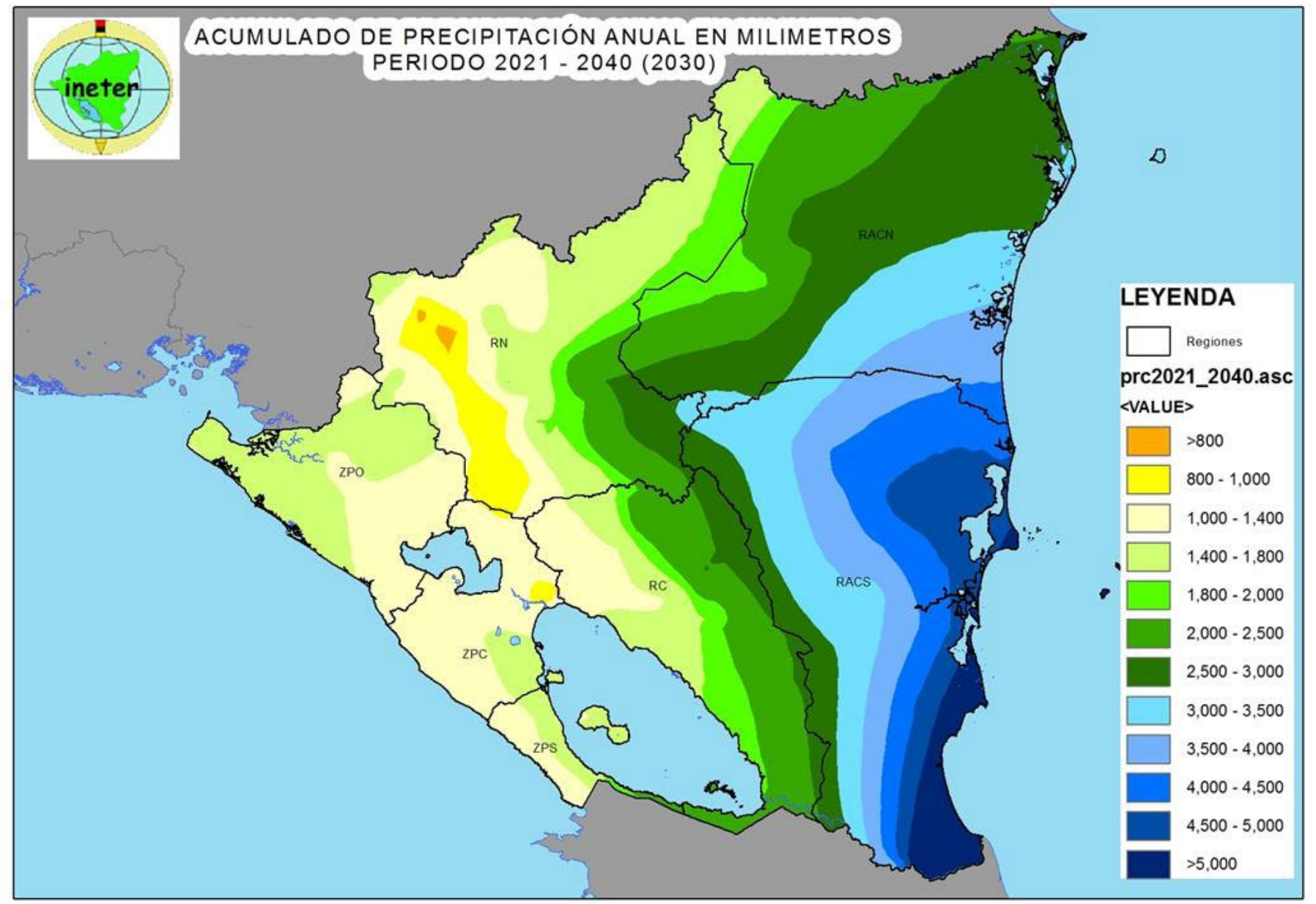

Figura 8. Evaluación de posibles escenarios de cambio climático en Nicaragua (INETER y MARENA, 2017).

Figure 8. Evaluation of possible scenarios of climate change in Nicaragua (INETER and MARENA, 2017).

- Creación/mejora del Sistema de Gestión de datos hidrológicos en los organismos de gestión, que incluyó la modelización de la interfaz gráfica para la carga y almacenamiento de datos y la construcción de una plataforma y base de datos para la recolección de información de pozos, piezómetros y manantiales en Argentina y Nicaragua.

- Incorporación del Proyecto RLA 7018 al Plan de Gestión de los Recursos hídricos de la Autoridad del Agua de la Provincia de Buenos Aires en Argentina, a la Agenda de Acciones de Gestión Integrada de Recursos Hídricos Superficiales y Subterráneos de la Agencia Nacional del Agua en Brasil, participación en la formulación del Plan Nacional del Agua en Ecuador con recomendaciones en aspectos específicos de las aguas subterráneas, fortalecimiento institucional, gestión integral para la protección y aprovechamiento de aguas subterráneas por cuencas hidrográficas y políticas de concienciación ciudadana, y recomendaciones para la validación y edición del mapa hidrogeológico del
Ecuador a escala 1:250.000, y contribución en la elaboración del Diagnóstico de los Recursos Hídricos y Desarrollo de Líneas Estratégicas del Ministerio del Ambiente y los Recursos Naturales en Nicaragua.

El Informe Final del Proyecto RLA 7018 (IAEA, 2018) consigna los logros generales alcanzados y detalla los resultados en cada área piloto.

EI Proyecto RLA 7024 ha comenzado el completado de vacíos técnicos e institucionales en sus áreas piloto, en particular se destacan las campañas de muestreo para determinación de isótopos estables, tritio, $14 \mathrm{C}$ y $13 \mathrm{C}$ y el fortalecimiento de la vinculación ente organismos de gobierno relacionados con los recursos hídricos y el ambiente.

\section{Principales lecciones aprendidas}

La experiencia adquirida en la ejecución de los proyectos RLA 7018 y 7024 permite esperar que las capacidades creadas estén disponibles en el futuro para asegurar la sostenibilidad de las acciones emprendi- 
das.

Durante la implementación de los proyectos se adquirieron las siguientes experiencias:

- Importancia de la participación de instituciones académicas que contribuyen en los proyectos a través de la generación de conocimiento científico, que ha sido transferido mediante el desarrollo de investigaciones de manera conjunta, apoyo en temas de tesis de postgrado, cursos de educación continua y cursos específicos ofrecidos a los profesionales de las instituciones relacionadas con los recursos hídricos.

- Importancia de la participación de instituciones responsables de la gestión de los recursos hídricos a nivel nacional / provincial y compromiso de sus más altas autoridades en la realización del proyecto. La sinergia entre las instituciones que tienen atribuciones en el ámbito de los recursos hídricos condujo a la identificación de manera consensuada de las brechas de conocimientos sobre hidrogeología a nivel nacional y al compromiso de superar estos vacíos, a la asignación de recursos humanos y económicos para la realización de campañas de muestreo para medición de parámetros fisicoquímicos y de isótopos estables y a dar continuidad al proyecto cuyos aspectos más importantes fueron incluidos en los Planes Nacionales o Provinciales de los Recursos Hídricos.

- Importancia de establecer alianzas efectivas en torno a propósitos comunes entre entidades que tienen entre sus funciones la gestión del recurso hídrico con criterios de sostenibilidad.

- Importancia de participar a un proyecto regional, intercambio de experiencias entre los países, de sus fortalezas y sus desafíos en materia de conocimiento y gestión de los recursos hídricos.

- Importancia del apoyo del OIEA para el lanzamiento del proyecto en cada país y de la asistencia técnica y logística durante el desarrollo del mismo.

\section{Sostenibilidad de la aplicación de la metodología IWAVE}

En las áreas piloto del Proyecto RLA 7018 se generaron iniciativas para implementar la metodología IWAVE en la gobernanza de los recursos hídricos mediante el fortalecimiento de estaciones hidrometeorológicas, a través de la adquisición de capacidades humanas e instrumentales y de aplicación de las técnicas isotópicas para los estudios de los recursos hídricos. Asimismo se crearon las bases para la sostenibilidad del proyecto mediante la generación de datos históricos, modelos conceptuales y numéricos del flujo y transporte subterráneo, balances hídricos, así como la ejecución de estudios de hidrología isotópica orientados a la GIRH.

En Argentina la Autoridad del Agua de la Provincia de Buenos Aires incluyó la aplicación de las técnicas isotópicas en su Plan de Gestión de los Recursos Hídricos con objeto de lograr una mejor comprensión de los procesos hidrológicos. En Brasil se utilizará un documento síntesis de los Estudios Hidrogeológicos y de Vulnerabilidad y Proposición de Modelo de Gestión Integrada y Compartida para elaborar términos de referencia para estudios específicos en dos cuencas del país, a fin de entender la relación de los cursos de agua superficiales con el Acuífero Urucuia, con el uso de isótopos y con apoyo del OIEA. En Ecuador se sentaron las bases para una gestión sostenible de los recursos hídricos subterráneos en las principales instituciones del país y existe el compromiso de dar continuidad al proyecto dado que los aspectos metodológicos fueron incluidos en el Plan Nacional del Agua. En Nicaragua el Gobierno Nacional promueve las coordinaciones estratégicas entre las instituciones que trabajan en el ámbito de los recursos hídricos y forman parte del equipo de gestión del proyecto, a fin de unir esfuerzos para explorar posibilidades de cooperación mediante la formulación de proyectos integrales y ha fijado su plan de acción a partir del Diagnóstico de los Recursos Hídricos y Desarrollo de Líneas Estratégicas.

En ambos proyectos, el RLA 7018 y RLA 7024, se aprecia el posicionamiento de la hidrología isotópica en los organismos de gestión como herramienta valiosa para mejorar la comprensión del funcionamiento de los sistemas hídricos.

\section{Conclusiones}

Los países de América Latina presentan algunas características similares y otras contrastantes, en cuanto a las brechas tecnológicas e institucionales, al estado de la gestión de los recursos hídricos subterráneos y a los requerimientos de capacitación.

La aplicación de IWAVE ha contribuido al fortalecimiento de las capacidades regionales para mejorar el conocimiento y la gestión de los recursos hídricos.

El rol de la investigación y la academia como generadora de conocimiento se articula al servicio de los objetivos misionales de las instituciones de decisión nacional en relación con la gestión de los recursos hídricos de forma integral.

Se espera que IWAVE ayude a los países a ser más eficientes y eficaces para resolver los problemas diferentes y específicos relacionados con los recursos hídricos. Una contribución significativa del proyecto es la mejora del conocimiento de las aguas subterráneas como parte de la planificación estratégica de cada país, lo que permitirá el desarrollo de una "hoja de ruta" a nivel nacional para la gestión de los recursos hídricos.

De esta manera, la comprensión hidrológica conduce a una mejor evaluación de la sostenibilidad de los recursos hídricos subterráneos en América Latina contribuyendo a su protección, gestión integrada y gobernanza. 


\section{Referencias}

Bocanegra, E., Toro Espitia, L., Araguas, L. 2015.The IWAVE methodology applied to improve the state of knowledge of water resources in Latin America. International Symposium on Revisiting Foundations and Exploring Frontiers, Isotope Hydrology, IAEA-CN-225, Viena, 1, 212-215.

Bocanegra, E., Toro Espitia, L., Araguas Araguas, L. 2016. Fortalecimiento de capacidades nacionales para mejorar la evaluación de los recursos hídricos subterráneos en América Latina. Revista Latinoamericana de Hidrogeología, 10 (1), 144 -152.

CEPAL. 2018. Proceso Regional de las Américas. Foro Mundial del Agua 2018. Informe Regional América Latina y el Caribe. Resumen ejecutivo.

https://www.cepal.org/sites/default/files/news/files/ informe_regional_america_latina_y_caribe.pdf, Acceso 20/05/2019

Dunning C. 2010. Water availability to be enhanced through focus on National Water Resources Assessments. IAEA. Water \& Environment News, 27, http://www-naweb.iaea.org/napc/ih/lHS_projects_ iwave.html, Acceso 20/05/2019

Gleick, P. 2017. Human right to water and global sustainability. Seminario Derecho Humano al Agua, Ciudad del Vaticano, 95-96. http://www.encuentromundi.org/wp-content/uploads/2017/12/Libro-Seminario-Derecho-Humano-al-Agua_Roma.pdf , Acceso 20/05/2019

IAEA. 2012. IWAVE The IAEA Water Availability Enhancement Project.

http://www-naweb.iaea.org/napc/ih/documents/ factsheetsPosters/IWAVE\%20leaflet.pdf, Acceso 20/05/2019

IAEA. 2013. IWAVE Helping Improve National Water Resources Management. Water \& Environment News. Newsletter of the Isotope Hydrology Section,
32, http://www.iaea.org/water, Acceso 20/05/2019

IAEA. 2018. Informe final del proyecto RLA/7/018 AR-

CAL CXXXV "Mejora del conocimiento de los recursos hídricos subterráneos para contribuir a su protección, gestión integrada y gobernanza."Informe Inédito, Sección de Hidrología Isotópica, IAEA, Viena, 60 págs.

INETER y MARENA. 2017. Atlas de Escenarios Climáticos de Nicaragua. Plataforma Nacional de Información y Conocimiento sobre Cambio Climático.

http://www.cambioclimatico.ineter.gob.ni/, Acceso $13 / 03 / 2019$

Mikhailova, N. 2019. Using isotopic techniques to map and analyse groundwater resources in the Sahel. Water Impact Stories, IAEA Bulletin, (4) 16-17.

PNUD. 2015. Objetivos de Desarrollo Sostenible.

http://www.undp.org/content/undp/es/home/sustainable-development-goals.html, Acceso 20/05/2019

Rives, D., Bocanegra, E. Quiroz Londoño, O.M., Ortega, L., Araguas, L. 2018. Mejora de la gestión de recursos hídricos subterráneos en la provincia de Buenos Aires a través del fortalecimiento de capacidades. El Agua Subterránea: Recursos sin Fronteras: Acuíferos Transfronterizos, El Agua Subterránea y las Ciudades, Planificación y Gestión, Universidad Nacional de Salta, Salta. (1) 171-178.

UN-WATER. 2015. Un Objetivo Global para el Agua Post-2015: Síntesis de las Principales Conclusiones y Recomendaciones de ONU - Agua. 46 págs.

http://www.un.org/spanish/waterforlifedecade/pdf/ findings_and_recommendations_post2015_goal_ water_spa.pdf, Acceso 20/05/2019

UNESCO. 2019. Informe Mundial de las Naciones Unidas sobre el Desarrollo de los Recursos Hídricos 2019 "No Dejar A Nadie Atrás". 198 págs. https:// unesdoc.unesco.org/ark:/48223/pf0000367304, Acceso 20/07/2019.Publicado: marzo 2021.
Recibido: julio 2019

Revisado: noviembre 2019

Aceptado: enero 2020

Publicado: marzo 2021 